\title{
长期航天飞行心血管保护: 问题与挑战
}

\author{
邢文娟 ${ }^{1,2}$, 邢长洋 ${ }^{1,3}$, 凌树宽 ${ }^{2}$, 李英贤 $2^{*}$, 高峰 ${ }^{1^{*}}$ \\ 1. 空军军医大学航空航天医学系, 西安 710032; \\ 2. 中国航天员科研训练中心, 航天医学基础与应用国家重点实验室, 北京 100094; \\ 3. 空军军医大学第二附属医院超声诊断科, 西安 710038 \\ *联系人, E-mail: yingxianli@aliyun.com; fgao@fmmu.edu.cn
}

收稿日期: 2021-01-27; 接受日期: 2021-04-12; 网络版发表日期: 2021-08-12

空间站工程航天医学实验项目(批准号: HYZHXM01001)、军队重大项目(批准号: AWS16J018)、军队重点基础研究项目(批准号: 2019-JCJQZD-196-00)、国家自然科学基金(批准号: $31670865,81701859,81822026$ )、陕西省高校科协青年人才托举计划项目(批准号: 20180302)、中国 博士后科学基金(批准号: 2020M673670)和空军军医大学凌云工程维鹰计划资助

摘要 我国即将运行的空间站要求航天员长期驻留太空, 航天飞行引发的心血管功能及循环系统调控改变直接 影响航天员的健康和工作效能, 其中失重是最重要的环境因素. 目前人们对短期航天飞行机体的生理变化及适应 规律已有一定的经验，但对长期失重所致的心血管变化规律、特征以及相关生理效应发生机制的认识还不够全 面和深入, 相应的心血管保护措施仍有待完善. 阐明及解决上述问题是我国载人航天发展战略的迫切需求. 本文 综述了失重/模拟失重条件下机体心血管系统的变化、适应特征及相关机制的国内外研究进展，尤其是针对空间 站长期微重力环境的心血管效应、现有心血管保护措施以及存在的问题与挑战进行了分析和讨论, 希望为我国 面向空间站任务长期航天飞行的心血管保护提供借鉴和新思路.

关键词长期航天飞行, 失重, 功能失调, 重塑, 心血管保护, 对抗措施

探索太空一直是人类的梦想. 1961年4月 12 日, 苏联 宇航员尤里·加加林驾驶“东方 1 号”宇宙飞船升入太空, 揭开了人类载人航天的序幕. 人类经过近 60 年的载人 航天历程，在航天员健康保障方面积累了宝贵的经验, 但长期航天和未来的星际飞行仍面临大量未知的风险, 一些重要的生物医学问题仍有待不断认识和探索. 失 重、电离辐射、幽闭环境及昼夜节律紊乱等是航天飞 行对健康的重要威胁 ${ }^{[1]}$. 其中, 失重所致的心血管功能 改变是影响航天员健康及工作效能的首要因素 ${ }^{[2,3]}$. 阐 明长期失重对心血管结构和功能的影响, 并研发有效
的对抗措施既是我国载人航天战略发展的需求, 也是 人类探索和认识太空环境对自身机能变化和适应能力 的迫切需要. 我国通过前期实际航天经历和地面模拟 微重力实验研究对失重产生的心血管效应已积累了一 些经验 ${ }^{[4]}$, 但面对空间站任务, 尤其是长期驻留太空对 心血管系统的远期影响方面仍有诸多问题尚不清楚. 本文在总结国内外关于失重心血管生理效应、适应机 制和对抗措施最新研究进展的基础上, 针对我国亟需 开展的长期载人航天飞行心血管效应及防护中的关键 科学和技术问题进行分析和展望，希望为我国空间站

引用格式: 邢文娟, 邢长洋, 凌树宽, 等. 长期航天飞行心血管保护: 问题与挑战. 中国科学: 生命科学, 2022, 52: 190-203

Xing W J, Xing C Y, Ling S K, et al. Cardiovascular protection in long-term spaceflight: current understanding and challenges (in Chinese). Sci Sin Vitae, 2022, 52: 190-203, doi: 10.1360/SSV-2020-0348 
及未来深空探测等长期载人航天医学保障提供参考.

\section{1 长期航天飞行与心血管功能失调}

失重是载人航天飞行中影响人体心血管功能和健 康的最重要因素, 其中立位耐力不良、运动能力降 低、心脏和血管结构及功能重塑是长期航天飞行导致 心血管系统功能失调的重要表现，这不仅是航天员舱 外活动的限制因素之一，还对返回地面时即刻的应急 离舱能力构成潜在威胁. 航天失重环境下机体各系统 变化有明显的时序性，且持续时间和反应程度亦不相 同。机体对失重环境也不断进行适应性调节，并逐渐 达到一种新的、适应失重环境的状态，即失重适稳态 $(\text { 图 } 1 \mathrm{~A})^{[5]}$. 航天员进入太空微重力环境, 首先出现血液
向头部转移, 循环系统很快发生一系列变化及相应适 应性调节和改变(图1).

\section{1 体液转移}

体液转移是航天飞行过程中循环系统变化的最重 要的始动因素. 机体进入微重力环境后, 随着重力和静 水压消失，动脉各部分的跨壁压分布和局部血流量发 生变化, 导致体液迅速转移和重新分布. 微重力引起 的体液转移主要有两种方式，一是下肢和腹部的体液 向中央和头部转移, 约有 $2 \mathrm{~L}$ 的体液从下半身转移至上 半身; 二是身体上部静脉压和毛细血管流体静压增加， 体液由毛细血管内向血管外转移 ${ }^{[6]}$.

体液转移和压力变化影响体液量及心血管结构和 功能. 进入微重力环境或模拟失重24 48 h内，血容量
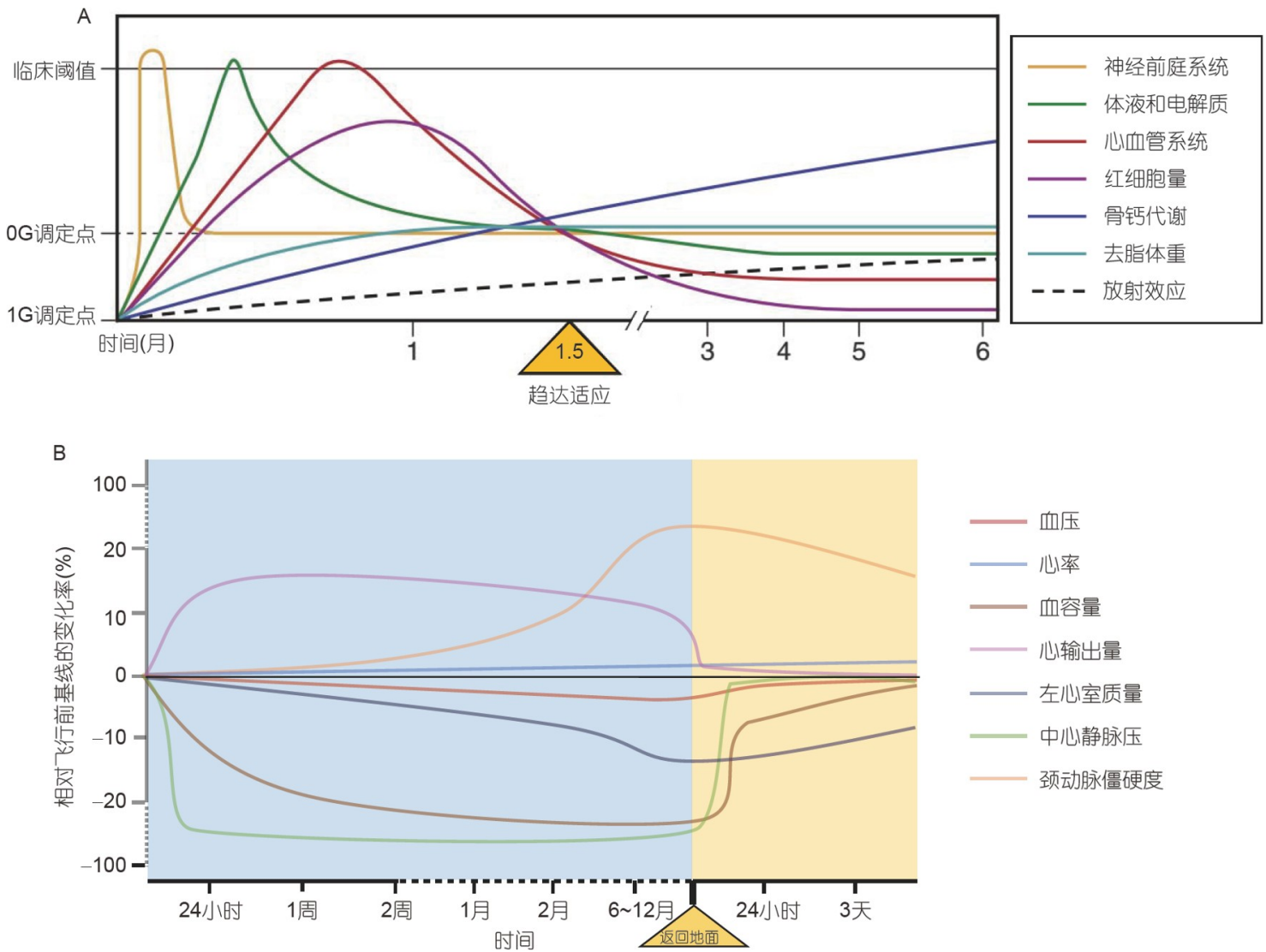

图 1 航天飞行过程中机体各系统的时序改变及循环系统主要指标的变化趋势. A：航天飞行中机体各系统时序改变(数据来 自http://asgsb.org/slidesets/slidesets.html)；B：航天飞行中及返回地面后的不同时间血容量、血压、心率、心输出量等各指标 发生变化的百分比(与飞行前坐位基准水平比较)(引自文献[7,12,15,16,52])

Figure 1 Temporal physiological responses in different systems during spaceflight and cardiovascular responses to microgravity during and after spaceflight in human. A: The temporal physiological responses to microgravity in different body systems (from http://asgsb.org/slidesets/slidesets. html); B: cardiovascular adaptive changes at different time points during and after spaceflight in comparison with pre-flight measurements taken in a seated posture (data from Refs. [7,12,15,16,52]). 
减少 $10 \% \sim 20 \%{ }^{[7]}, 3 \sim 5$ 天内达到平衡. 血容量减少的主 要机制包括: 毛细血管前后阻力改变、毛细血管对体 液的通透增加使体液向血管外转移; 心脏扩大和体液 向上半身的重分布刺激颈动脉压力感受器, 导致神经体液因素改变; 液体摄入量减少等 ${ }^{[8,9]}$. 通过对数次航 天飞行前、中、后的超声心动图数据分析发现，进入 太空初期由于血液向中心转移致回心血量快速增加, 心脏体积增大，而后随着对失重环境的适应心脏体积 逐渐缩小，最终小于地面基础值. 与在地面立位相比, 航天飞行早期血液向中心转移导致心肺血容量增加, 每搏量和心输出量均明显增加(图1B $)^{[10,11]}$.

\section{2 心脏结构和功能变化}

失重及模拟失重致心脏结构重塑和/或质量改变 的现象在人和大小鼠等均有报道. 磁共振成像显示, 航 天飞行 10 天后航天员心肌质量降低了 7\% 10\% ${ }^{[12]}$. 长 期失重致心脏萎缩的机制目前尚不完全清楚，可能与 重力负荷降低所致心脏的容积和压力负荷减少、体力 活动减少和代谢需求降低等的适应性改变有关. 心脏 萎缩与血容量减少、长期回心血量下降共同导致了心 脏储备能力的减弱, 加之骨骼肌萎缩, 从而影响航天员 运动能力和工作效能. 目前在航天飞行中每搏量和心 输出量测量的个体差异较大．前苏联“礼炮7号”及“联 盟”载人飞船 237 天长期航天飞行的超声心电图检测结 果显示，飞行2 3个月后航天员多出现左室舒张末容 积、收缩末容积和每搏量减少，飞行近 8 个月后，2名 航天员左室舒张末容积持续降低(14\%, 29\%), 每搏量 降低 $(15 \%, 12 \%)$. 近些年几项研究结果观察到 $3 \sim 12$ 个 月航天飞行中的心输出量增加，与早期国际空间站及 俄罗斯空间站上观测到的心输出量降低或不变 ${ }^{[13,14]}$ 并 不一致，例如，Norsk等人 ${ }^{[15]}$ 的报道及2018年美国航空 航天局(National Aeronautics and Space Administration, NASA) 进行的为期一年的双胞胎航天对照研究 (NASA Twins Study). 在NASA Twins Study研究中, 航 天员Scott Kelly在国际空间站航天飞行并进行了飞行 前、中、后监测，其同卵双生兄弟作为遗传匹配的地 面对照. 该研究发现，航天员在空间站飞行期间的心 输出量比地面直立位时平均增加 $10 \%{ }^{[16]}$. 心输出量测 量差异的主要原因可能在于飞行前地面检测体位和方 法的不同. 通常在地面仰卧位较直立位或坐位所测心 输出量基础值高 $15 \%$ 29\%. 有报道显示，与地面仰卧
位相比，航天飞行期间心输出量和每搏量分别下降 $11.9 \%$ 和 $9.4 \%$, 而与地面坐位相比则心输出量增加 $10 \%$ 。 心输出量较地面坐位/立位的升高及失重状态下 的体液转移, 增加了头颈部血量, 进而促进航天飞行 相关的神经-眼综合征(spaceflight associated neuro-ocular syndrome, SANS)的发生. 据NASA报道, 国际空间 站上工作的航天员有 $50 \%$ 以上存在相关的眼部症状.

由于地面立位/坐位的心输出量受到重力本身作 用的影响，因此太空飞行期间与地面仰卧位基线的对 比，可更准确地反映失重环境下心血管系统的功能状 态和变化. 我们在模拟失重的尾吊大、小鼠上均发现, 4周的模拟失重即可引起心肌质量减少、心肌应变(收 缩功能)下降、心脏舒张功能降低(二尖瓣舒张早期/晚 期血流速度比值 $E / A<1)(\text { 图2 })^{[17]}$, 且右心和左心呈现不 同程度的重塑和恢复速度 ${ }^{[18]}$. 这与早期国际空间站及 俄罗斯空间站上观测到的心输出量改变相似，提示这 种以轻度心脏充盈减少、心肌萎缩、心脏做功减少 ${ }^{[8]}$ 为特征的“太空心”是长期失重所致的心脏主要变化.

心脏萎缩、血容量减少和回心血量下降、心脏收 缩/舒张功能的受损共同导致返回地球后直立位心输 出量显著降低，这也是航天员返回地面时发生立位耐 力不良(orthostatic intolerance)的主要原因之一. 虽然 目前NASA采取的防护措施(运动锻炼和返回前体液 加载)可以较为有效地防止航天飞行期间的心脏功能 下降 ${ }^{[19]}$, 但由于国际空间站驻留时间一般为 6 个月, 目 前关于更长时间航天飞行对心脏影响的认识尚有限, 尤其是长期航天飞行所致的心脏结构和功能重塑在返 回地面后是否逐渐恢复或在一段时间内持续加重，以 及这些重塑改变对远期心脏健康的影响仍有待进一步 明确.

\section{3 血管重塑和功能失调}

微重力状态下，流体静压的消失使人体不同部位 血管内压发生不同的变化，产生相应的适应性结构重 塑与功能改变. Arbeille等人 ${ }^{[20]}$ 报道, 6 个月太空飞行使 航天员颈动脉内中膜厚度增加 $10 \%$ 12\%; NASA Twins Study亦发现，为期 1 年的航天飞行使颈动脉内中膜厚 度增加了约 $20 \%$, 返回 4 天后亦未恢复 ${ }^{[16]}$. 我国学者张 立藩课题组 ${ }^{[21,22]}$ 通过模拟失重尾吊大鼠不同部位动脉 结构和功能的系统性研究证实, 心脏以上血管出现肥 厚性结构改变，心脏以下出现血管萎缩、收缩反应降 



图 2 模拟失重4周致大鼠心肌萎缩、心脏收缩及舒张功能减低. 与对照相比, 模拟失重4周后大鼠左心室质量(LVM)减低, 心 肌应变绝对值(收缩功能)下降、同步性降低(不同节段时间差增加)，二尖瓣舒张早期/晚期血流速度比值(舒张功能)下降(作者 待发表数据). MD, 机械弥散性指数

Figure 2 Cardiac atrophy and declined systolic and diastolic function induced by 4-week simulated microgravity in rats. Hindlimb unloading for 4 weeks led to decreased left ventricular mass (LVM), reduced absolute value of global longitudinal strain (GLS) (systolic function) and mitral Doppler flow E/A (diastolic function), and increased mechanical dispersion (myocardial dyssynchrony) (unpublished data). MD, mechanical dispersion

低和血管周围神经支配减弱的变化，即动脉系统发生 “区域差异性重塑”; 并提出航天飞行后心血管功能失 调的“心血管外周效应器机制”假说, 认为失重条件下 动脉血管平滑肌-内皮-管周神经共同形成的“外周效 应器”结构重塑和功能变化是航天飞行后心血管系统 功能失调的重要原因. 本课题组的研究显示, 模拟失重 4 8周尾吊大鼠脉搏波传播速度(pulse wave velocity, PWV)显著增加, 大动脉顺应性下降. 而大量临床研究 显示，脉搏波传播速度增加与老龄及高血压、脑卒中 等心血管事件密切相关. 上述血管内膜中层增厚和脉 搏波传播速度增加是否是一种适应性的可逆变化及其 与长期航天飞行后远期心脑血管疾病风险的关系目前 尚不清楚. 此外, 失重也引起静脉血管区域性重塑及下 肢静脉顺应性增加, 使返回重力场后立位时血液在下 肢滞留增加，有效回心血量减少，这可能也是航天飞 行后立位耐力下降的原因之一.

与上半身其他动脉 (如颈动脉)相似，模拟长期失 重还可导致大鼠脑动脉出现收缩反应增强与肥厚性重 塑的变化. “礼炮6号”空间站飞行过程中曾观察到航天 员脑血管反应性的改变. 航天员飞行后进行 $-30^{\circ}$ 头低 位倾斜试验，显示脑血管收缩反应较飞行前增强，且 该变化在失重环境停留的时间越长则越明显, 这一效 应的恢复时间也越长 ${ }^{[23]}$; 脑血管有完善的自身调节机 制, 当动脉血压在60 140 mmHg范围内变动时, 脑血 管的自身调节机制可使脑血流量保持相对稳定. 虽然 失重所致的静水压和血压的改变可能对脑血管的自身
调节机制没有产生直接影响，然而长期航天飞行脑血 管自身调节功能仍可能发生改变, 从而促使立位耐力 不良发生 ${ }^{[24]}$. 研究表明, 卧床2周后受试者脑循环的自 身调节能力下降 ${ }^{[25]}$, 脑动脉与脑血流动力学发生变 化 ${ }^{[26]}$. NASA Twins Study显示, 太空飞行持续12个月 对认知能力具有一定负面影响 ${ }^{[16]}$; 本课题组在尾吊大 鼠实验中观察到, 模拟长期失重可促发脑血管衰老样 改变, 并可能加速脑结构及认知功能发生退行性改变.

\section{4 心律与心血管调节功能变化}

在美国开展空间项目的早期, 出舱活动、锻炼及 下体负压应用等过程中航天员均进行心电检测. 1977 年报道的首例因心脏疾病返回地面的原因就是心律紊 乱; “礼炮 7 号”空间站 1 名苏联航天员也因出舱活动中 发生间歇性心律失常而提前返回. 在“和平号”10年的 飞行任务中, 共观察到31例异常心电图和75例心律失 常, 其诱因不仅有失重, 还包括血钾降低、心理生理 应激、睡眠不良等.

1991年，美国在航天飞机上首次用颈套法加压和 减压刺激颈动脉压力感受器, 发现反射增益及心电R$\mathrm{R}$ 间期均低于飞行前，提示航天员颈动脉窦压力感受 器在失重环境下敏感性降低. 这可能是航天员在返回 重力场后产生立位耐力降低的重要原因之一. Hughson等人 ${ }^{[27]}$ 对 6 名男性航天员在国际空间站2 6个月飞 行任务中心率变异性(heart rate variability, HRV)的分 析显示, 飞行中和飞行后高频频谱功率降低, 提示副交 
感神经兴奋降低，而压力反射功能在飞行中基本稳定; 返回地面后，心率略有加快，压力反射功能降低 $25 \%$ $\sim 34 \%$. 这些结果表明, 通过现有运动训练等措施干预 已基本可以满足航天员中长期空间在轨飞行心血管功 能维持正常，返回地面后依然存在轻度的心血管调节 功能失调. 此外, 长期航天飞行的航天员返回时出现 血液循环中肾上腺素水平升高，但心血管对肾上腺素 的响应却减弱 ${ }^{[28]}$, 提示心血管的神经-体液调节变化可 能是立位耐力不良发生的重要因素.

因此，失重导致心血管功能失调涉及系统调节的 多个环节，血容量降低、颈动脉窦压力感受器敏感性 下降、下肢静脉顺应性增加及肌肉萎缩等都参与其发 生, 且随着航天员在轨飞行时间的延长, 这些不良影响 可能会更严重.

\section{2 长期失重/模拟失重心血管改变的分子 机制}

微重力环境下, 细胞是否可直接感受重力变化及 其机制，特别是细胞是否存在感受重力变化的特异细 胞器或重力感应结构是目前国际航天医学领域关注的 一个重要科学问题. 已知细胞膜及膜蛋白、细胞骨架、 胞外基质等都可感受机械力的变化, 尤其是膜流动性是 细胞膜的基本特征之一并受重力影响, 这可能是细胞感 知重力的基本机制之一 ${ }^{[29]}$. 细胞膜上存在力学信号的 传感器(如机械力敏感的钙通道Piezo)、生长因子受体 等. 失重引起的细胞外力学信号变化, 通过这些感受分 子可转化为细胞膜自身通道活性变化或下游信号通路 变化, 从而将力学信号传递至细胞内 ${ }^{[30,31]}$. Kapitonova等 人 ${ }^{[32]}$ 研究了 12 天航天飞行对离体人脐静脉内皮细胞 (human umbilical vein endothelial cells, HUVECs)的直接 作用, 发现细胞膜通透性增加、细胞骨架明显损伤、线 粒体数量减少、细胞代谢和增殖能力降低.

钙作为细胞内第二信使，也在失重状态的信号调 控中发挥重要作用. 失重可影响细胞膜上机械力敏感 的钙通道活性, 对细胞钙稳态和细胞功能发挥重要影 响. 本课题组 ${ }^{[33]}$ 发现，小鼠心肌HL-1细胞在模拟失重 模型中自发性钻振荡增加、胞浆钙浓度均升高，细胞 内钙的增加导致钙调蛋白依赖的蛋白激酶 II / 组蛋白 去乙酰化酶4(calmodulin-dependent protein kinase II/ histone deacetylase 4, CaMKII/HDAC4)信号的激活, 病
理性心肌重塑信号分子HDAC4的激活促发了心肌细 胞重塑(图3). 在抛物线飞行实验中发现，力学变化可 诱导心肌细胞内钙浓度升高, 该效应主要由 $\mathrm{T}$ 型钻通 道激活增加从而诱导细胞内钻释放所介导 ${ }^{[34]}$. 模拟长 期失重条件下大鼠脑血管平滑肌细胞 $\mathrm{T}$ 型钙通道 Cav3.1上调, 通过调节钻调神经磷酸酶(calcineurin)/ NFATc3通路促进细胞从收缩表型向增殖表型转换，导 致脑血管结构重塑 ${ }^{[35]}$. 尾吊56天心肌细胞雷诺丁受体 (RyR) 磷酸化水平升高导致了细胞钙信号异常, 可能 是失重性心律不齐的原因之一 ${ }^{[36]}$.

重力对细胞基因表达、蛋白翻译和翻译后修饰及 稳定性也具有重要影响. NASA科研人员在空间站上 观察到果蝇肌原纤维及细胞外基质(extracellular matrix, ECM)重构, 并通过转录组分析发现微重力条件 下细胞外基质基因和蛋白酶体调节基因发生了差异表 达, 提示微重力诱导心肌细胞蛋白稳态的改变 ${ }^{[37]}$. 本 课题组发现酪蛋白激酶2相互作用蛋白 1 (casein kinase-2 interacting protein-1, CKIP-1)缺失促进HDAC4 出核和转录因子MEF2转录活性增加, 进而促进心肌重 塑的发生 ${ }^{[38]}$, 尾吊模拟失重条件诱导小鼠心肌CKIP-1 mRNA和蛋白表达降低，是导致其病理性心肌重塑发 生的原因之一 ${ }^{[39]}$.

\section{3 失重的心血管保护与对抗措施}

采取有效的对抗失重措施可明显减轻太空飞行对 航天员心血管的不良影响, 并有助于返回地面后的再 适应. 理论上, 在航天器内建立人工重力环境是对抗 失重影响的最理想方案 ${ }^{[21]}$, 其途径有两种, 一是整个 航天器旋转产生“连续性人工重力”, 二是舱内短臂离 心机旋转产生“间断性人工重力”. 前者从设计到实 施、操作存在诸多困难; 后者相对较为可行, 但航天 飞行中间断性人工重力的大小、时间和频率以及工程 安装等仍存在较多有待解决的问题.

针对失重导致的心血管功能紊乱的防护，目前采 用的措施主要包括主动运动、辅助设备防护、药物防 护以及飞行前的适应性训练等(图4).

\section{1 运动锻炼}

运动锻炼是对抗多种“航天病”的最重要措施. 目 前载人航天中最常用的方法是自行车功量计和跑台. 


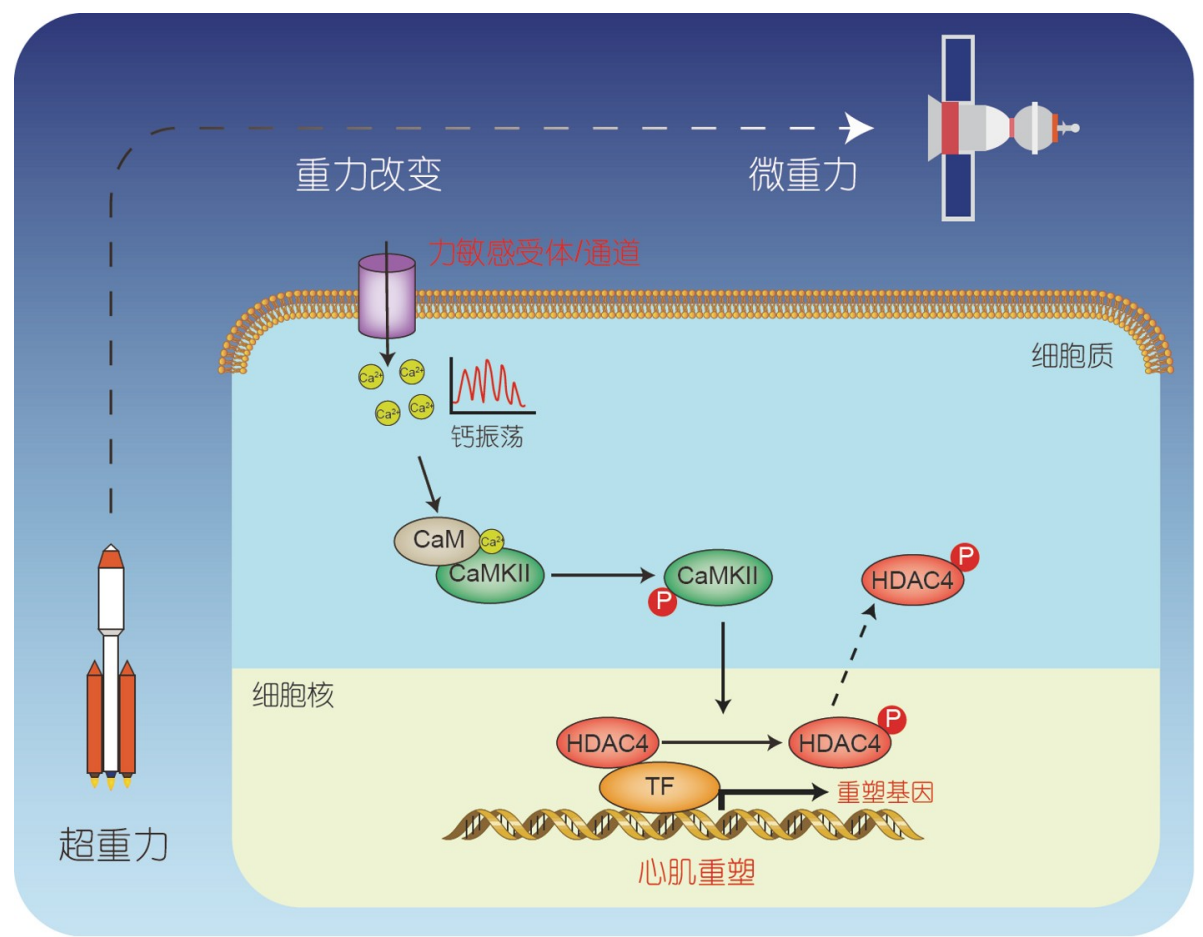

图 3 模拟失重条件下心肌细胞重构的钙信号机制. 重力环境改变使心肌细胞自发性钲振荡增加、胞浆钙浓度升高，导致钙 调蛋白依赖的蛋白激酶 II/组蛋白去乙酰化酶4信号激活, 促发重塑基因表达和心肌重构(改编自文献[33])

Figure 3 Schematic of calcium signaling mechanism underlying the effects of simulated microgravity on myocardial remodeling. Alterations of gravity induce dynamic changes in spontaneous calcium oscillations and intracellular calcium signaling. The increase in [C $\left.{ }^{2+}\right]_{i}$ activates $\mathrm{CaMKII} /$ HDAC4 signaling pathway and induces expression of remodeling genes and myocardial remodeling (adapted from Ref. [33])

主要机制

血容量降低
心肚重塑
血管重塑
静脉顺应性增加
脑血管自身调节能力降低
压力感受器敏感性降低
自主神经调节失调
骨骼肌萎缩



立位耐力不良
对抗措施

运动锻炼

企鹅服

本液加轰

范荷服

人工重力

图 4 立位耐力不良发生的主要机制和目前采用的对抗措施. 对抗措施中, 运动锻炼和企我服为在轨每天应用措施; 体液加载 和抗荷服为返回前一次性使用措施; 下体负压、药物、营养饮食和人工重力为辅助或尚处于研究阶段的防护措施

Figure 4 Mechanisms of post-flight orthostatic intolerance and countermeasures. Exercise training and Penguin suit are daily in-flight countermeasures; fluid and salt loading and anti-G garment are used at the end of the flight before landing; lower body negative pressure, pharmacological agents, nutrition supplement and artificial gravity are supplementary countermeasures still under research and development

长期航天飞行中重力负荷的降低及舱内幽闭环境航天 员活动减少，很快导致心血管负荷及代谢改变、肌萎 缩、骨丢失等，运动可增加心血管负荷并调动和增加
有效循环血量，提高心肺功能和有氧工作能力，同时 也可维持下肢肌力, 对抗失重性肌萎缩和代谢改变. 运动锻炼既“生理”又相对简单易行，尤其是效果确切, 
因而一直备受重视. 由于运动不仅占用航天员在轨飞 行大量宝贵的时间, 且消耗航天器内较多生命支持物 资，因此，选择高效的运动方式、制定最佳的运动方 案是国际载人航天健康保障的一个重要问题. Hughson等 ${ }^{[27]}$ 的研究表明，在轨飞行期间运动训练对维持 心血管适应航天环境非常重要，目前通用的每天 $2.5 \mathrm{~h}$ 在轨有氧运动训练能有效维持长期航天飞行心血管系 统功能稳定. 近年国际载人航天飞行中航天员的运动 方案也在不断改进和完善 ${ }^{[40],}$ 除传统的有氧运动外, 抗阻锻炼也被应用于航天运动方案中，其主要对下肢 肌群力量和耐力有较好的锻炼效果. 由于不同航天任 务飞行时间不同及航天员个体差异，长期载人航天运 动锻炼的具体方案仍是各航天大国积极探索并致力完 善的重要问题，其宗旨是尽可能简易、舒适、高效地 对抗各种“航天病”. 中国航天员科研训练中心 2019 年 开展并完成了“地星二号” 90 天头低位卧床实验，旨在 探寻失重条件下跑步锻炼、抗阻锻炼及多种锻炼措施 的运动防护效果及最佳组合方案，为空间站航天员长 期在轨飞行健康保障提供更全面的科学依据. 未来针 对在轨运动锻炼措施的进一步细化研究, 包括各类型 锻炼的最佳运动量和运动频次, 运动效果在轨评估和 调整等, 将为航天员提供更有效和个性化的运动保护 方案.

\section{2 药物}

药物防护具有简单、省时、省事的优点，在航天 员健康防护中也一直备受关注. 调节心血管系统功能 的药物, 如复方甘油和脉律定(防止心律失常)、抗利 尿激素和加压素(防止水和电解质紊乱)、调节自主神 经的药物(提高立位耐力)等都曾被美国和俄罗斯航天 员使用.

中医药是中华医学的瑰宝, 其“整体观”“辨证施 治”的理论及中药多成分、多作用靶点和多作用途径 的特点, 使之在航天医学中可能发挥独特的作用, 因而 具有特殊潜在的价值. 我国李勇枝课题组 ${ }^{[41]}$ 开展的航 天生命保障系统中药防护措施研究显示, 服用中药“太 空养心丸”可明显改善60天头低位卧床期间的心脏射 血和收缩功能，对脑血流和立位耐力不良也有一定的 改善作用. 本课题组发现, 西洋参的主要有效成分 ——西洋参茎叶总㿝苷可缓解模拟失重大鼠心肌萎 缩、改善射血分数 ${ }^{[42]}$; 并对骨内 $\mathrm{H}$ 血管 $\left(\mathrm{CD} 31^{\mathrm{hi}} \mathrm{Emcn}{ }^{\mathrm{hi}}\right.$
的血管亚型)具有一定的保护效果，防止失重性骨丢 失 $^{[43]}$. 这些传统中药经中医辨证评估、药理、药代明 确和防护方案确定后，有望为航天员健康和心血管保 护提供具有中国特色的更佳保障措施.

\section{3 辅助设备}

航天飞行期间, 航天员穿着“企我服”, 在进行操作 活动和运动时须克服服装弹力作用, 以此达到锻炼肌 肉的效果. 此外, 可通过在航天员腰部以下施加一定 的负压，促使血液向下肢转移，通过人为改变血液分 布以对抗失重引起的血流动力学改变. 采用的主要设 备包括负压筒、下体负压裤、自行下体负压训练器 等. 航天员从太空返回地面时和返回后穿着抗荷服, 可 以减少重力作用所致的下肢血液淤积，有助改善和提 高立位耐力.

\section{4 其他措施}

航天员返回地面前的体液加载(口服补液盐水)可 快速增加血容量, 是减轻返回过程和返回后立位耐力 下降的经典方法. 但该措施扩容效果并不理想，仅对 短期航天飞行引起的立位耐力下降有一定缓解 ${ }^{[44]}$. 研 究发现, 卧床试验中给受试者补充蛋白质可减轻心脏 萎缩 ${ }^{[45]}$. 本课题组 ${ }^{[17]}$ 最近发现, 时间限制性饮食(timerestricted feeding, TRF)可改善模拟长期失重大鼠心肌 糖代谢和心脏功能，提示时间限制性饮食有望成为长 期航天飞行一种新的心脏保护措施. 此外, 航天员返 回地面后有针对性地采用营养、理疗、低负荷力量与 耐力训练等综合康复措施, 可促进其心血管功能和运 动能力恢复, 使其尽快重新适应地球重力, 并提高其 再次参加飞行任务训练的能力.

综上，运动锻炼是目前长期航天飞行中保护心血 管功能最有效、最常用的措施 ${ }^{[46]}$, 通过饮食和营养调 节改善代谢也是安全、可行的方法，航天飞行期间及 返回地面后多重综合防护措施的联合应用仍是最稳 妥、可靠的策略.

\section{4 问题与挑战}

目前人们对长期失重所致的心血管效应的认识 和经验还很有限, 对其发生机制和适应规律等科学 问题的研究还不够深入和全面, 相应的心血管保护 
措施仍有待进一步探索和完善. 面对空间站任务及 未来的星际飞行，航天员是否还有未知的健康风险 以及还存在哪些新的医学保障问题等均有待阐明. 其中已知较重要的科学和技术问题主要包括以下7 方面.

\section{1 长期航天飞行立位耐力下降及其对抗措施}

航天飞行后返回地面或进入其他重力场会出现立 位耐力不良, 即突然受到重力作用导致回心血量及心 脏每搏量显著降低, 加之前期失重所致心血管调节和 代偿能力不足, 难以维持直立位血压, 轻者出现头 晕、心率增快, 重者几天不能站立, 立位时发生晕厥. 早期航天飞行实践表明，航天员返回地面无一例外地 出现不同程度的立位耐力降低. 长期航天飞行 (129 190天)约有 $83 \%$ 的航天员返回地面时出现立位 耐力不良，明显高于短期飞行的发生率 $(20 \% \sim 30 \%)^{[28]}$. 对抗立位耐力不良一直是航天医学的一个重要问题及 难题. 近年来随着防护措施的改进, 严重立位耐力不良 的发生率明显下降 ${ }^{[47]}$. 最新研究显示, 国际空间站的 运动方案和体液加载已可有效减轻返回地球 $24 \mathrm{~h}$ 内的 日常活动中的立位耐力不良 ${ }^{[19]}$. 但已有的单一对抗措 施尚不足以完全对抗心血管功能失调, 仍需要综合运 用多种措施减轻返回地球或未来登月、登火后立位耐 力不良的发生.

\section{2 长期航天心血管改变的特点及对返回地面后 航天员远期健康的影响}

目前认为, 失重所致的多数心血管功能性改变是 可逆的. 本课题组 ${ }^{[18]}$ 在长期失重尾吊动物发现, 颈动 脉的脉搏波传播速度增加和心室重塑在尾吊结束后 可在很大程度上逐步恢复, 但长期微重力所致血管和 心脏的结构及相应的功能改变是否能完全恢复, 以及 具体多久及什么程度的结构和功能改变是可恢复的 阈值等尚不清楚. 此外, 长期航天飞行暴露的微重力 环境, 包括不可忽视的辐射环境等均可能对心血管产 生不可逆的影响, 如加速冠状动脉原本难以察觉的早 期病理改变或加重不良后果. 尤其是关于长期航天飞 行对返回地面后远期心血管疾病的发病风险的影响 目前仍有不同观点. Hamilton等人 ${ }^{[48]}$ 通过计算Framingham风险评分, 评估了美国航天员全因心脏疾病 风险，发现无论男性还是女性航天员其40 50岁时发
生心血管疾病的风险为 $3 \% \sim 5 \%$; 参与过阿波罗登月 计划的宇航员心血管疾病死亡率较未参与任何太空 飞行的宇航员高4 5倍 ${ }^{[49]}$, 提示太空飞行可能对远期 心血管健康存在不良影响. Ade 等人 ${ }^{[50]}$ 的多因素风险 模型评价分析显示, NASA的310名宇航员与981名 NASA非宇航员雇员对照相比心血管疾病发病风险无 显著差异; 短期航天飞行并未显著增加航天员心肌梗 死、充血性心衰、脑卒中等严重心血管疾病的发病 风险. 2019年美国、俄罗斯、法国联合的一项队列研 究报道, 一名航天员在国际空间站飞行约 2 个月后出 现颈静脉血栓，55\%的航天员出现颈静脉血液瘀滞甚 至反流 ${ }^{[51]}$, 这无疑会增加航天飞行中或飞行后发生脑 血栓、肺栓塞等严重心血管事件的风险. 此外, 许多 心脑血管疾病都与血管损伤或功能下降密切相关, 而 长期失重条件下血管老化或损伤的发生是否影响长 远的心血管健康仍不清楚. 因此, 除注重研发更有效 的心血管保护措施外，还亟需开发和完善更敏感的检 测技术，争取早期预警和发现心脏、脑血管、心脏冠 状动脉或其他血管潜在病变.

\section{3 长期航天飞行促发血管衰老样改变的特点、 机制及特异性干预措施}

新近研究显示, 长期航天飞行造成的心血管改变 是一种“衰老样”改变, 包括体能下降、心输出量降 低、动脉硬化、胰岛素抵抗等 ${ }^{[9]}$. 弹性动脉僵硬度增 加、顺应性下降、内膜增厚、舒张功能下降以及血 管修复/新生能力降低等是衰老血管的重要特征. Hughson等人 ${ }^{[52]}$ 一项对 8 名航天员的研究发现，6个月 的航天飞行后航天员颈动脉偠硬度增加 $17 \%$ 30\%, 其血管老化的程度相当于地面 10 20年的正常增龄改 变; 重要的是, 除颈动脉外股动脉动脉傈硬度也明显 增加, 提示除了体液转移直接导致上半身血管内压增 加外, 还存在全身影响因素, 其中血浆超氧化物歧化 酶(superoxide dismutase, SOD)、基质金属蛋白酶-2 (matrix metalloproteinase-2, MMP2)等的增加及全身 胰岛素敏感性的降低均可能参与上述血管改变. 动脉 偪硬度增加与心脏病和卒中等心血管事件风险及阿 尔茨海默症风险的增加密切相关, 因此长期航天飞行 的血管保护值得特殊关注. 尤其是长期航天飞行可能 存在的心血管代谢适应不良逐渐引起各国重视, 如加 拿大国家航天局(Canadian Space Agency, CSA)血管 
健康系列研究 (Vascular Series) 拟持续开展 15 年 (2009 2024年)，通过检测航天员飞行前、中、后的 不同时间各部位动脉僵硬度变化、运动血流变化及 胰岛素敏感性、钲代谢等，以进一步明确血管衰老发 生以及返回地面 1 年后的恢复情况和变化机制(www. revibiomedica.sld.cu/index.php/ibi/rt/printerFriendly/ 250/385). 此外, NASA Twins Study以及之后更多航天 员的研究发现, 航天飞行重返地面后外周血单核细胞

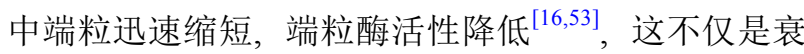
老的标志，也提示了衰老相关心血管疾病的潜在风 险. 本课题组在大鼠尾吊和猕猴头低位卧床的研究均 显示，长期模拟失重可促进颈、脑血管衰老样改变, 内皮依赖性舒张功能降低, 并影响脑代谢和认知功 能, 其中血管平滑肌细胞机械力感受器Piezo1及 $\mathrm{miR}$ NAs介导的代谢变化在模拟失重引发的血管衰老样 改变中可能发挥重要作用(未发表数据). 因此, 血管保 护可能是长期太空飞行需要特别关注的一个重要问 题. 进一步明确长期失重对不同血管的结构、功能和 代谢的影响, 尤其是其可能的促血管衰老发生的特点 和机制及对心脑等重要靶器官的潜在影响, 并探寻相 应的防护措施, 是目前航天医学亟待阐明的一个重要 问题.

\section{4 长期航天飞行对心血管影响的性别和个体差 异及其机制}

航天飞行对心血管系统的影响在不同性别中的差 异越来越受到重视. 目前我国进行的地面模拟失重人 体头低位卧床试验大多在男性受试者中开展，大鼠、 小鼠尾吊实验也大多使用雄性. 事实上，性别的差异 在失重所致心血管功能失调中确实存在，如女性比男 性更易发生直立性晕厥，且体液损失更显著等 ${ }^{[54]}$; 而 男性更易产生由于乑内血液循环、脑脊液循环动力学 改变引发的SANS而影响视力 ${ }^{[55]}$. 此外, 航天员 6 个月 航天飞行产生胰岛素抵抗在男性中更明显 ${ }^{[52]}$. 因此对 抗措施产生的效果可能也有差别，如有报道，人工重 力对抗立位耐力不良的效果在男性要优于女性 ${ }^{[56]}$. 此 外，人类对太空飞行的生理反应和对抗效应往往也存 在不同程度的个体差异. 因此，进一步深入认识长期 失重所致心血管效应的性别差异和个体差异将为有针 对性地制定精准失重对抗方案，实现特性化防护提供 理论依据.

\section{5 航天生理效应研究模型和技术限制问题}

航天医学研究最棘手的问题是航天员人数少且难 以建立严格对照, 而地面模拟失重研究缺乏理想的动 物实验模型. 目前地面模拟失重的人体试验主要是头 低位卧床法和浸水法 ${ }^{[57,58]}$, 动物实验主要是采用大鼠 小鼠头低位悬吊模型, 细胞实验主要是利用回转器模 拟失重. 然而地面模型所模拟的效应与实际航天飞行 仍有一定差距, 例如, 非直立行走动物(大鼠、小鼠)对 尾吊头低位的敏感性低 ${ }^{[57]}$; 其中后肢去负荷、体液转 移及头颈部血管静水压增大等效应可被较好模拟，而 心血管重塑的特点，如心肌萎缩程度则不如航天飞行 显著 ${ }^{[59]}$. 令人欣慰的是, 我国即将建成的载人空间站 是未来国际重要的空间研究实验室, 将为失重的生理 效应和机制研究提供可靠的研究平台. 因此，充分利 用好我国即将建成的载人空间站进行研究, 可突破地 面研究的局限性, 并验证地基研究结果, 将极大的推 动我国重力生物学、航天生理学和航天医学等相关领 域的研究进展.

另一方面，航天在轨研究的技术条件也有其局限 性. 空间飞行环境的特殊性使得地面上较为成熟的研 究平台和技术常常难以直接应用于航天飞行过程，技 术操作、实验控制、实验对象数量、样本保存等也可 能在一定程度上限制获得生理数据的可靠性, 例如, 心 输出量的检测目前采用的有无创外源气体重呼吸法、 连续指脉波波形分析法、超声检测法和颈动脉舒张直 径估算法等，这些方法的原理、敏感度和精度各不相 同，所得结果差异较大. 而近年多尺度建模及人工智 能(artificial intelligence, AI) 等技术的进步有助于人们 认识复杂且难以直接检测的一些生理变量的估算及趋 势预测，如基于多尺度建模可综合分析失重条件下血 流动力学变量和心脏功能, 包括飞行时间的影响、全 身不同部位血管和血流速度改变等 ${ }^{[60]}$. 今后航天飞行 心血管医学监测和研究中有待开发及令人期待的技术 包括: 先进的三维实时超声成像技术检测心脏结构; 血 流动力学、自主神经活动和激素水平的同步监测; 下 体负压和运动过程中动态心血管功能监测; 耐受性较 好的 24 h动脉血压监测; 太空飞行环境下的体温变化 精准检测; 血管内皮功能的无创检测和超声血管弹性 (脉搏波传导速度等)及脑血流的在轨检测; 远程超声 机器人系统(robotically controlled ultrasound system); 
人工智能辅助实时在轨生理测评, 获取高质量、多维 度的心血管健康多维信息以进行早期风险评估; 集成 化航天医学诊疗和空-地远程诊疗等. 因此, 在空间站 上建立更加完善且简单易行的在轨生理评价技术和体 系将极大地促进长期航天飞行的医学、生理学研究和 医学保障.

\section{6 揭示心血管改变及适应的分子机制, 实施特性 化的精准对抗措施}

近半个世纪以来的航天医学研究主要着重于太空 环境下机体的整体效应和系统调控, 但目前对于航天 生理效应发生机制和对抗防护措施的研究主要围绕某 个组织或器官开展. 而机体作为一个整体, 各个组织器 官之间始终是相互作用、共同协调维持着动态平衡并 不断适应环境改变. 如失重条件下神经-内分泌-免疫应激反应 ${ }^{[61]}$ 或代谢状态改变 ${ }^{[17]}$ 与心血管系统的交互 调控; 空间飞行过程中肠道菌群的多样性 ${ }^{[62]}$ 及其代谢 变化对心血管系统的共同调节等. 因此一方面对于不 同组织器官间、系统间的协同调控作用, 特别是太空 环境下以心血管系统为核心的多种生理适应变化和调 控规律尚有待深入研究; 另一方面, 还要深入认识和揭 示微重力条件下机体调控及适应的分子机制, 以制定 更高效、精准的防护措施, 因此航天分子医学相关的 基础研究尤显重要.

近年方兴未艾的组学(-omics)技术以及单细胞测 序技术的发展极大地提升了人类获取生物信息的广度 和速度, 可以帮助人们全方位更深入理解重力因素对 细胞、器官乃至对人体的影响, 多角度分析航天飞行 对机体的影响规律和多层次的复杂调控机制 ${ }^{[63]}, 2019$ 年美国《科学》专刊报道的NASA Twins Study将基因 组学、转录组学、蛋白组学、代谢组学以及延伸的表 观基因组学、表观转录组学的数据整合分析, 是NASA 开展的第一项整合组学研究 ${ }^{[16]}$. 应用上述技术, 这项 “ $n=1$ ” 的航天员研究获得了大量珍贵的数据, 为航天医 学研究及长期航天医学保障提供了重要信息. 2020年 《细胞》及其子刊《细胞报告》报道了航天医学的系 列研究最新进展, 通过对 28 名航天员的多组学研究提 示航天飞行返回后肌肉再生与炎症反应相关, 为个性 化药物治疗提供了潜在靶点和标志分子 ${ }^{[64]}$. 此外, NASA研究人员通过对小鼠组织或人类细胞的数百个 样本(NASA GeneLab数据库)的转录组学、蛋白组学、
代谢组学和表观遗传学数据分析, 发现线粒体相关生 物过程的显著变化; 来自NASA Twins Study和国际空 间站其他三个人体研究项目的 59 名航天员血尿样本的 生物医学资料也显示线粒体功能改变和DNA损伤的证 据，提示线粒体应激和功能改变可能是太空多种生物 效应的核心机制 ${ }^{[65]}$. 这些研究及其带来的启示将有助 于人们对长期飞行的失重生理效应开展精准对抗防护 并建立更加完善的生命支持系统, 也将使人们对特殊 环境、极端环境生理变化规律有更深入的认识.

\section{7 航天综合环境因素对心血管影响的协同效应}

我国即将运行的载人空间站要求确保航天人员 180 天以上的驻留能力. 长期太空飞行除失重外, 应 激、幽闭环境、昼夜节律改变、太空微生物、电离辐 射等特殊环境因素对航天员健康的影响是整体的综合 效应(图5), 这些特殊因素的共同作用对心血管系统的 影响更加复杂. 长期应激可造成机体免疫功能降低, 幽 闭环境对心理的影响巨大; 太空昼夜节律的改变可引 起神经-内分泌功能失调, 加重心理及睡眠障碍, 并与 失重引起的脑血流灌注改变共同影响航天员的认知及 心血管调控能力, 成为长期航天飞行影响航天员心血 管健康和任务执行能力的重要因素. 此外, 在未来探 月、登火等星际飞行任务中, 太空辐射对健康的影 响、尤其是对血管内皮细胞损伤和促炎效应更显重 要. 辐射通过增加氧化应激、线粒体功能障碍和细胞 衰老等机制加速动脉弹样硬化的进展、增加缺血性心 脏病的发病风险, 且与微重力效应产生协同作用, 因此 在未来星际飞行中辐射对心血管系统的影响及其防护 也应引起特殊关注 ${ }^{[9]}$.

\section{5 总结}

我国载人航天“三步走”战略即将迈入第三步，进 入空间站时代，建立大型、长期有人照料的空间站指 日可待. 目前我国已成功经历了六次载人航天飞行实 践, 然而与美国、俄罗斯相比, 我国开展有规模的载 人空间探索活动以及基于载人空间活动的科学研究起 步较晚, 长期载人航天研究尚处于初级阶段 ${ }^{[66]}$, 仍有 一些重大医学问题亟待解决. 虽然截至目前的研究表 明，短期航天飞行对心血管系统未造成严重不可逆的 影响, 说明机体生命做关的器官经过长期进化具有强 


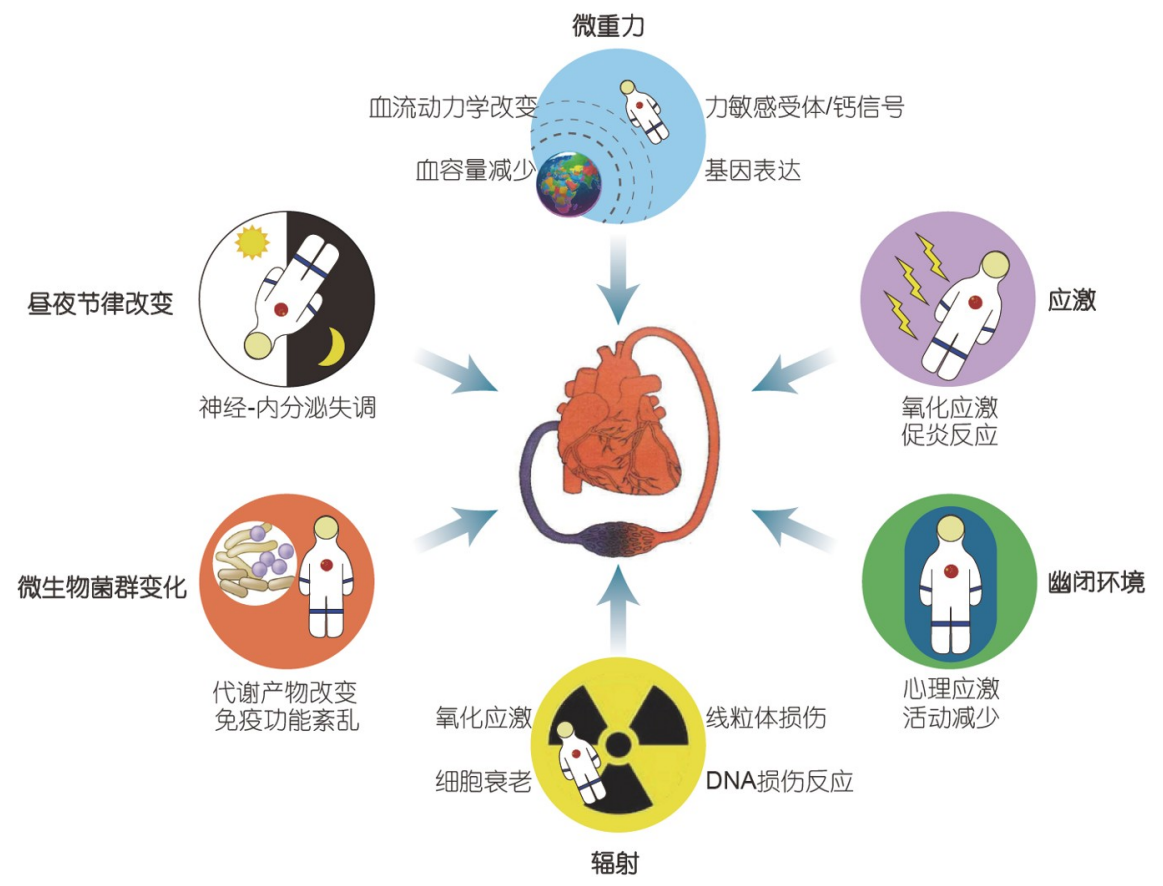

图 5 航天综合环境对心血管系统的影响. 航天飞行中微重力、辐射、幽闭环境、昼夜节律改变、应激、微生物菌群变化等 多种环境因素共同作用, 导致心血管结构和功能发生适应性改变或损伤

Figure 5 Impact of comprehensive space environment on cardiovascular system. Microgravity, ionizing radiation, isolated environment, circadian desynchrony, stress, gut microbiome shifts and other spaceflight hazards impact cardiovascular health, leading to cardiovascular structural and functional adaptive changes or impairments

大功能储备和适应能力，辅以现有的对抗措施能够基 本应对近地轨道变重力的挑战. 但是长期太空飞行所 面临的未知潜在风险，以及长期慢性适应是否会导致 适稳态超负荷而对心血管健康造成远期影响尚不清 楚．进一步获得航天员空间中长期太空驻留的系统性 健康数据，明确太空各种因素对心血管系统等的影响，
并研发更有效的综合防护和对抗措施是我们工作的重 要目标. 深入认识长期航天心血管功能改变及其适应 规律, 建立精准的在轨生理检测技术和评价体系以及 个性化、高效的心血管保护策略，将为我国面向空间 站的长期航天飞行生命保障和健康支持体系提供有力 支持, 并为人类空间探索提供重要生物医学基础.

致谢感谢空军军医大学航空航天医学系王䶍博士后和中国航天员科研训练中心航天医学基础与应用国家重点实验 室杜锐凯老师对本文提出的宝贵建议并帮助绘制插图.

\section{参考文献}

1 Lobrich M, Jeggo P A. Hazards of human spaceflight. Science, 2019, 364: 127-128

2 Li Y H. Current status and trend of space medicine researches (in Chinese). Space Med Med Eng, 2013, 26: 421-425 [李荣辉. 航天医学研究现 状与趋势. 航天医学与医学工程, 2013, 26: 421-425]

3 Chen S G, Deng Y B, Li Y H. Major progresses and prospects of space medico-engineering (in Chinese). Space Med Med Eng, 2018, 31: 79-89 [陈善广, 邓一兵, 李莹辉. 航天医学工程学主要研究进展与未来展望. 航天医学与医学工程, 2018, 31: 79-89]

4 Wang K, Gao F. Review and prospects of fifty years' development of environment control and life support system in manned spacecraft (in Chinese). Space Med Med Eng, 2011, 24: 435-443 [王康, 高峰. 载人航天器环控生保系统50年研制回顾与展望. 航天医学与医学工程. 2011, 24: 435-443]

5 Gao F, Yu M S. Homeostasis and allostasis (in Chinese). Acta Physiol Sin, 2020, 72: 677-681 [高峰, 俞梦孙. 稳态与适稳态. 生理学报, 2020, 


\section{2: 677-681]}

6 Watenpaugh D E, Buckey J C, Lane L D, et al. Effects of spaceflight on human calf hemodynamics. J Appl Physiol, 2001, 90: 1552-1558

7 Arbeille P, Gaffney F A, Beck L, et al. Effect of microgravity on renal and femoral flows during LBNP \& intravenous saline load. J Gravit Physiol, 1996, 3: 91-92

8 Watenpaugh D E. Fluid volume control during short-term space flight and implications for human performance. J Exp Biol, 2001, 204: 32093215

9 Hughson R L, Helm A, Durante M. Heart in space: Effect of the extraterrestrial environment on the cardiovascular system. Nat Rev Cardiol, 2018, 15: 167-180

10 Tank J, Jordan J. Mighty hearts in space. J Physiol, 2015, 593: 485-486

11 Xing C Y, Liu Y N, Xing W J, et al. Physiological compensatory mechanisms of improved left heart function in response to simulated acute weightlessness: a human echocardiographic study by speckle tracking strain imaging (in Chinese). Sci Sin Vitae, 2021, 51: 190-198 [邢长洋, 刘] 云楠, 邢文娟, 等. 模拟急性失重致左心功能代偿提高及其调节特性一人体超声斑点追踪应变研究. 中国科学: 生命科学, 2021, 51: 190198]

12 Perhonen M A, Franco F, Lane L D, et al. Cardiac atrophy after bed rest and spaceflight. J Appl Physiol, 2001, 91: 645-653

13 Herault S, Fomina G, Alferova I, et al. Cardiac, arterial and venous adaptation to weightlessness during 6-month MIR spaceflights with and without thigh cuffs (bracelets). Eur J Appl Physiol, 2000, 81: 384-390

14 Hamilton D R, Sargsyan A E, Martin D S, et al. On-orbit prospective echocardiography on international space station crew. Echocardiography, 2011, 28: 491-501

15 Norsk P, Asmar A, Damgaard M, et al. Fluid shifts, vasodilatation and ambulatory blood pressure reduction during long duration spaceflight. J Physiol, 2015, 593: 573-584

16 Garrett-Bakelman F E, Darshi M, Green S J, et al. The NASA Twins Study: A multidimensional analysis of a year-long human spaceflight. Science, 2019, 364: eaau8650

17 Wang X P, Xing C Y, Zhang J X, et al. Time-restricted feeding alleviates cardiac dysfunction induced by simulated microgravity via restoring cardiac FGF21 signaling. FASEB J, 2020, 34: 15180-15196

18 Zhong G, Li Y, Li H, et al. Simulated microgravity and recovery-induced remodeling of the left and right ventricle. Front Physiol, 2016, 7: 274

$19 \mathrm{Fu}$ Q, Shibata S, Hastings J L, et al. Impact of prolonged spaceflight on orthostatic tolerance during ambulation and blood pressure profiles in astronauts. Circulation, 2019, 140: 729-738

20 Arbeille P, Provost R, Zuj K. Carotid and femoral artery intima-media thickness during 6 months of spaceflight. Aerosp Med Hum Perform, 2016, 87: 449-453

21 Zhang L F. Region-specific vascular remodeling and its prevention by artificial gravity in weightless environment. Eur J Appl Physiol, 2013, 113: 2873-2895

22 Zhang L F, Hargens A R. Spaceflight-induced intracranial hypertension and visual impairment: Pathophysiology and countermeasures. Physiol Rev, 2018, 98: 59-87

23 Gazenko O G, Genin A M, Egorov A D. Summary of medical investigations in the U.S.S.R. manned space missions. Acta Astronaut, 1981, 8: $907-917$

24 Zuj K A, Arbeille P, Shoemaker J K, et al. Impaired cerebrovascular autoregulation and reduced $\mathrm{CO}_{2}$ reactivity after long duration spaceflight. Am J Physiol Heart Circ Physiol, 2012, 302: H2592-H2598

25 Zhang R, Zuckerman J H, Pawelczyk J A, et al. Effects of head-down-tilt bed rest on cerebral hemodynamics during orthostatic stress. J Appl Physiol, 1997, 83: 2139-2145

26 Sun X Q, Yao Y J, Yang C B, et al. Effect of lower-body negative pressure on cerebral blood flow velocity during 21 days head-down tilt bed rest. Med Sci Monit, 2005, 11: CR1-CR5

27 Hughson R L, Shoemaker J K, Blaber A P, et al. Cardiovascular regulation during long-duration spaceflights to the international space station. J Appl Physiol, 2012, 112: 719-727

28 Meck J V, Reyes C J, Perez S A, et al. Marked exacerbation of orthostatic intolerance after long- vs. short-duration spaceflight in veteran astronauts. Psychosom Med, 2001, 63: 865-873

29 Kohn F, Hauslage J, Hanke W. Membrane fluidity changes, a basic mechanism of interaction of gravity with cells? Microgravity Sci Technol, 
2017, 29: 337-342

30 Lewis A H, Grandl J. Mechanical sensitivity of Piezo1 ion channels can be tuned by cellular membrane tension. eLife, 2015, 4: e12088

31 Ranade S S, Syeda R, Patapoutian A. Mechanically activated ion channels. Neuron, 2015, 87: 1162-1179

32 Kapitonova M Y, Muid S, Froemming G R, et al. Real space flight travel is associated with ultrastructural changes, cytoskeletal disruption and premature senescence of HUVEC. Malays J Pathol, 2012, 34: 103-113

33 Liu C, Zhong G, Zhou Y, et al. Alteration of calcium signalling in cardiomyocyte induced by simulated microgravity and hypergravity. Cell Prolif, 2020, 53: e12783

34 Acharya A, Brungs S, Lichterfeld Y, et al. Parabolic, flight-induced, acute hypergravity and microgravity effects on the beating rate of human cardiomyocytes. Cells, 2019, 8: 352

35 Zhang B, Chen L, Bai Y G, et al. miR-137 and its target T-type $\mathrm{Ca}_{\mathrm{V}} 3.1$ channel modulate dedifferentiation and proliferation of cerebrovascular smooth muscle cells in simulated microgravity rats by regulating calcineurin/NFAT pathway. Cell Prolif, 2020, 53

36 Respress J L, Gershovich P M, Wang T, et al. Long-term simulated microgravity causes cardiac RyR2 phosphorylation and arrhythmias in mice. Int J Cardiol, 2014, 176: 994-1000

37 Walls S, Diop S, Birse R, et al. Prolonged exposure to microgravity reduces cardiac contractility and initiates remodeling in drosophila. Cell Rep, 2020, 33: 108445

38 Ling S, Sun Q, Li Y, et al. CKIP-1 inhibits cardiac hypertrophy by regulating class ii histone deacetylase phosphorylation through recruiting PP2A. Circulation, 2012, 126: 3028-3040

39 Ling S, Li Y, Zhong G, et al. Myocardial CKIP-1 overexpression protects from simulated microgravity-induced cardiac remodeling. Front Physiol, 2018, 9: 40

40 Wang L J, Li Z L, Liu B K. Strategy considerations for countermeasures to weightlessness physiological effects in long-term spaceflight (in Chinese). Space Med Med Eng, 2012, 25: 442-448 [王林杰, 李志利, 刘炳坤. 长期航天飞行失重生理效应防护策略分析. 航天医学与医学工 程, 2012, 25: 442-448]

41 Li Y Z. Application of Chinese medicine and herbs in manned spaceflight (in Chinese). Space Med Med Eng, 2008, 21: 198-205 [李勇枝. 中医 药在载人航天中的应用. 航天医学与医学工程, 2008, 21: 198-205]

42 Sun H, Ling S, Zhao D, et al. Panax quinquefolium saponin attenuates cardiac remodeling induced by simulated microgravity. Phytomedicine, 2019, 56: 83-93

43 Liang S, Ling S, Du R, et al. The coupling of reduced type H vessels with unloading-induced bone loss and the protection role of Panax quinquefolium saponin in the male mice. Bone, 2021, 143: 115712

44 Platts S H, Tuxhorn J A, Ribeiro L C, et al. Compression garments as countermeasures to orthostatic intolerance. Aviat Space Environ Med, 2009, 80: $437-442$

45 Dorfman T A, Levine B D, Tillery T, et al. Cardiac atrophy in women following bed rest. J Appl Physiol, 2007, 103: 8-16

46 Navasiolava N, Yuan M, Murphy R, et al. Vascular and microvascular dysfunction induced by microgravity and its analogs in humans: Mechanisms and countermeasures. Front Physiol, 2020, 11: 952

47 Aubert A E, Larina I, Momken I, et al. Towards human exploration of space: The theseus review series on cardiovascular, respiratory, and renal research priorities. NPJ Microgravity, 2016, 2: 16031

48 Hamilton D R, Murray J D, Kapoor D, et al. Cardiac health for astronauts: Current selection standards and their limitations. Aviat Space Environ Med, 2005, 76: 615-626

49 Delp M D, Charvat J M, Limoli C L, et al. Apollo lunar astronauts show higher cardiovascular disease mortality: Possible deep space radiation effects on the vascular endothelium. Sci Rep, 2016, 6: 29901

50 Ade C J, Broxterman R M, Charvat J M, et al. Incidence rate of cardiovascular disease end points in the national aeronautics and space administration astronaut corps. J Am Heart Assoc, 2017, 6: e005564

51 Marshall-Goebel K, Laurie S S, Alferova I V, et al. Assessment of jugular venous blood flow stasis and thrombosis during spaceflight. JAMA Netw Open, 2019, 2: e1915011

52 Hughson R L, Robertson A D, Arbeille P, et al. Increased postflight carotid artery stiffness and inflight insulin resistance resulting from 6-mo spaceflight in male and female astronauts. Am J Physiol Heart Circ Physiol, 2016, 310: H628-H638

53 Luxton J J, McKenna M J, Lewis A, et al. Telomere length dynamics and DNA damage responses associated with long-duration spaceflight. Cell 
Rep, 2020, 33: 108457

54 Kusumbe A P, Ramasamy S K, Adams R H. Coupling of angiogenesis and osteogenesis by a specific vessel subtype in bone. Nature, 2014, 507: 323-328

55 Platts S H, Bairey Merz C N, Barr Y, et al. Effects of sex and gender on adaptation to space: Cardiovascular alterations. J Womens Health, 2014, 23: 950-955

56 Evans J M, Knapp C F, Goswami N. Artificial gravity as a countermeasure to the cardiovascular deconditioning of spaceflight: Gender perspectives. Front Physiol, 2018, 9: 716

57 Watenpaugh D E. Analogs of microgravity: Head-down tilt and water immersion. J Appl Physiol, 2016, 120: 904-914

58 Amirova L, Navasiolava N, Rukavishvikov I, et al. Cardiovascular system under simulated weightlessness: Head-down bed rest vs. Dry immersion. Front Physiol, 2020, 11: 395

59 Morey-Holton E, Globus R K, Kaplansky A, et al. The hindlimb unloading rat model: Literature overview, technique update and comparison with space flight data. Adv Space Biol Med, 2005, 10: 7-40

60 Gallo C, Ridolfi L, Scarsoglio S. Cardiovascular deconditioning during long-term spaceflight through multiscale modeling. npj Microgravity, 2020, 6: 27

61 Cao D, Song J, Ling S, et al. Hematopoietic stem cells and lineage cells undergo dynamic alterations under microgravity and recovery conditions. FASEB J, 2019, 33: 6904-6918

62 Liu Z, Luo G, Du R, et al. Effects of spaceflight on the composition and function of the human gut microbiota. Gut Microbes, 2020, 11: 807-819

63 Li Y X, Ling S K, Zhao Y L, et al. Advances in cellular and molecular basics of space medical problems (in Chinese). Space Med Med Eng, 2018,31: 140-151 [李英贤, 凌树宽, 赵亚丽, 等. 航天医学问题的细胞分子基础研究进展. 航天医学与医学工程, 2018, 31: 140-151]

64 Gertz M L, Chin C R, Tomoiaga D, et al. Multi-omic, single-cell, and biochemical profiles of astronauts guide pharmacological strategies for returning to gravity. Cell Rep, 2020, 33: 108429

65 da Silveira W A, Fazelinia H, Rosenthal S B, et al. Comprehensive multi-omics analysis reveals mitochondrial stress as a central biological hub for spaceflight impact. Cell, 2020, 183: 1185-1201.e20

66 Shang P, Hu Y T, Yang Z Q, et al. Tendency and critical scientific issues of space life science in China (in Chinese). Sci Sin Tech, 2015, 45: 796808 [商澎, 呼延霆, 杨周岐, 等. 中国空间生命科学的关键科学问题和发展方向. 中国科学: 技术科学, 2015, 45: 796-808]

\title{
Cardiovascular protection in long-term spaceflight: current under- standing and challenges
}

\author{
XING WenJuan ${ }^{1,2}$, XING ChangYang ${ }^{1,3}$, LING ShuKuan $^{2}$, LI YingXian ${ }^{2}$ \& GAO Feng ${ }^{1}$ \\ 1 School of Aerospace Medicine, Fourth Military Medical University, Xi'an 710032, China; \\ 2 State Key Laboratory of Space Medicine Fundamentals and Application, China Astronaut Research and Training Center, Beijing 100094, China; \\ 3 Department of Ultrasound Diagnostics, Tangdu Hospital, Fourth Military Medical University, Xi'an 710038, China
}

China's space station Tiangong is expected to be completed and will operate in the low-Earth orbit in the near future. During the longduration flight in space, microgravity is one of the major hazards that affect crew members' long-term cardiovascular health and task performance. The profound effects of microgravity on cardiovascular adaptation during long-term spaceflight and the underlying mechanisms have not been fully understood, which hampers the development of effective cardiovascular protective countermeasures. Here, we review and discuss the current research advances concerning long-term microgravity-induced cardiovascular responses, adaptations and possible underlying mechanisms. We further highlight and discuss the perspectives and challenges in developing more efficient cardiovascular protective countermeasures for long-term spaceflight.

long-term spaceflight, microgravity, dysfunction, remodeling, cardiovascular protection, countermeasures

doi: $10.1360 /$ SSV-2020-0348 